\title{
TRASTORNOS MUSCULOESQUELÉTICOS EN PROFESORES: ESTUDIO DE ENFERMERÍA DEL TRABAJO
}

\author{
MUSCULOSKELETAL DISORDERS IN TEACHERS: \\ OCCUPATIONAL HEALTH NURSING STUDY
}

\author{
Marta Regina Cezar-Vaz \\ Marlise Capa Verde de Almeida ${ }^{* *}$ \\ Laurelize Pereira Rocha ${ }^{* * *}$ \\ Anelise Miritz Borges ${ }^{* * *}$ \\ Luana de Oliveira Severo ${ }^{* * * * *}$ \\ Clarice Alves Bonow ${ }^{* * * * *}$
}

\begin{abstract}
RESUMEN
Este estudio tuvo como objetivo identificar las características del trabajo y sus implicaciones en la aparición de trastornos musculoesqueléticos en profesores. Este es un estudio descriptivo y exploratorio, realizado en el marco del proyecto de investigación "Salud, Riesgos y Enfermedades Ocupacionales - estudio integrado en diferentes ambientes de trabajo". Para la recolección de los datos se utilizó un cuestionario que contempló variables relacionadas a la caracterización de los sujetos, aspectos ambientales de la escuela y de los trastornos osteomusculares. La población del estudio fue de profesores representantes de 27 escuelas en un municipio de Río Grande do Sul. El manejo de los datos se realizó a través de estadísticas descriptivas. Las características del entorno de trabajo que más se destacaron fueron: espacio insuficiente en el aula (59,5\%)e inadecuación de los muebles que se utilizan en el desarrollo del trabajo (51,4 \%). Las patologías más incidentales fueron el dolor lumbar $(54,1 \%)$ y el dolor dorsal $(56,8 \%)$. Los resultados permitieron la identificacion de los síntomas y de las patologías entre todos los participantes investigados, sugiriendo, según el autorreporte de las profesoras, la posible influencia de las características y las condiciones del trabajo en la ocurrencia de los trastornos musculoesqueléticos. Se constata el conocimiento adquirido como indicativo de que el/la enfermero/a puede actuar frente a los riesgos y pérdidas que afectan a grupos específicos de profesionales, como los profesores.
\end{abstract}

Palabras clave: Salud laboral, trastornos de traumas acumulados, enfermería del trabajo, profesores.

\begin{abstract}
The aim of this study is to know the working features and the implications in the occurrence of osteomuscular disorders in teachers. This is a descriptive and exploratory study, conducted under the research project "Health, Risk and Illnesses - Integrated study in different work environments". For data collection a questionnaire that included variables related to the characterization of subjects, aspects of the school environment and musculoskeletal disorders. The study's population was of teachers who represented 27 schools in a city in Rio

\footnotetext{
* Enfermera. Profesora Escola de Enfermagem, Universidade Federal do Rio Grande, Brasil. Coordinadora del Laboratorio de Estudios de Procesos Socioambientales y Producción Colectiva de Salud - LAMSA. Email: cezarvaz@vetorial.net

${ }^{* *}$ Enfermera. Estudiante de doctorado, Universidade Federal do Rio Grande, Brasil. Email: marlisealmeida@msn.com

${ }^{* * *}$ Enfermera. Estudiante de doctorado, Universidade Federal do Rio Grande, Brasil. Email: laurinharoch@hotmail.com

${ }^{* * * *}$ Enfermera. Estudiante de doctorado, Universidade Federal do Rio Grande, Brasil. Email: miritzenfermeira@yahoo.com.br

${ }^{* * * * *}$ Enfermera. Estudiante de maestría, Universidade Federal do Rio Grande, Brasil. Email: luanasevero.enf.@gmail.com

${ }^{* * * * * *}$ Enfermera. Profesora Universidade Federal do Pampa, Brasil. Email: claricebonow@unipampa.edu.br
} 
Grande do Sul. The data handling was done through descriptive statistics and theme analysis. Work environment characteristics that stood out were: not enough space in the classroom $(59,5 \%)$ and inadequacy of the furniture used in the development of work $(51,4 \%)$. The most incident pathologies were low back pain $(54,1 \%)$ and back pain $(56,8 \%)$. The results permitted the identification of symptoms and pathologies among all the researched participants, thus emerging the teachers self-report, the possible influence of features and teaching working conditions in the occurrence of osteomuscular disorders. The knowledge acquired elucidates a field of nursing for easing risks and losses that suffer specific professional groups, such as teachers.

Key words: Occupational health, cumulative trauma disorders, occupational health nursing, teachers.

Fecha recepción: 12/01/13 Fecha aceptación: 29/07/13

\section{INTRODUCCIÓN}

Los ambientes de trabajo presentan características particulares que influyen en la salud de los trabajadores. Entre ellas, se destaca el esfuerzo muscular/esquelético corporal, exigido en las actividades rutinarias desarrolladas por profesores de Educación Infantil y Enseñanza Fundamental. Estudios previos (1-13) han señalado este compromiso en esa clase trabajadora, que converge para el desencadenamiento de Lesiones por Esfuerzos Repetitivos (LER) y Enfermedades Osteomusculares Relacionadas al Trabajo (EORT). Las referidas patologías pueden ser consideradas como una de las principales lesiones relacionadas al trabajo, afectando nervios, músculos y tendones, especialmente en miembros superiores (9-10, 14-15).

En relación con aspectos organizacionales, las quejas musculoesqueléticas pueden estar atribuidas a las condiciones de infraestructura del ambiente, organización y sobrecarga de trabajo que, muchas veces, es ejercido en posiciones ortostáticas no confortables, en función de los largos períodos de pie, durante el ejercicio de las actividades laborales: escribir en el pizarrón, cargar materiales didácticos para el aula, ejecutar la instalación de recursos audiovisuales y moverse constantemente de un edificio a otro. Además de estos factores, también contribuyen la carencia de local adecuado para descanso y la inadecuación de mesas y sillas, instrumentos indispensables para ejercicio del trabajo docente $(1,11,16,17)$.

Según estudios (5), un gran porcentaje de profesores acude a los servicios de salud con quejas osteomusculares, remitiendo la posibilidad de incapacidad para el trabajo, visto que las patologías señaladas se caracterizan como una de las principales causas de alejamiento ocupacional, identificado no solamente entre la clase profesional investigada, sino también entre otras categorías, como es el caso de los trabajadores de la salud (1819), trabajadores portuarios (20), bancarios (21-22), dentistas (23) y trabajadores rurales (24-28).

Los profesores que desarrollaron actividades altamente exigentes buscando cambios en las metodologías de enseñanza y en el rol del profesor en la sociedad presentaron perjuicios en su salud que generaron licencias médicas y ausentismo laboral (29).

Su origen multicausal dificulta la identificación del factor desencadenante específico, pues, en la mayor parte de los casos patológicos, las causas ultrapasan los síntomas físicos, incorporando también factores inherentes a la organización del trabajo, dificultades interpersonales y factores ergonómicos. Más allá de eso, la identificación de síntomas físicos causadores de dolores específicos en miembros y articulaciones hace que el individuo comience, automáticamente, a evitar movimientos y esfuerzos que reflejen dolor en ese segmento del miembro, enmascaran- 
do la sintomatología hasta extinguir ciertas actividades rutinarias, debido al progreso de la enfermedad y a la intensificación de los síntomas, dificultando el diagnóstico de la patología (14).

Con eso, se vuelve evidente la necesidad de establecer un nexo entre los factores que envuelven el trabajo docente y las características capaces de generar daños musculo-esqueléticos a los profesionales en foco. Entre las posibilidades de actuación clínica de la enfermería en esa realidad están la anamnesis ocupacional, el examen clínico, la asociación de la ciencia de la enfermería con informes médico-asistenciales y la educación en salud, tendiendo a instrumentalizar el quehacer del profesional enfermero para el suministro de recomendaciones preventivas, previstas en la legislación en salud del trabajador (29).

En el referido ámbito de trabajo, son más evidentes las legislaciones centradas en la dinámica educativa, de forma que el profesor atienda los requisitos propuestos por los órganos gubernamentales de educación. Pero para ello se debe enfocar en los factores ocupacionales que faciliten el buen desempeño del profesor, y es en esa dirección que la enfermería puede hacer uso de instrumentos de trabajo capaces de ampliar su actuación en el área de salud del trabajador, insertándose en el contexto para reconocer las actividades de riesgo y prevenir el surgimiento de sintomatologías patológicas.

Por las razones expuestas, se justifica la relevancia de la temática para la salud del trabajador y para el planeamiento de acciones preventivas por parte de los profesionales de la salud, en especial de el/la enfermero/a. Se vuelve necesaria la claridad de los aspectos ocasionadores de los mencionados agravios a la salud, desde la perspectiva de los profesores, para así presentar estrategias de minimización de los efectos lesivos de las enfermedades osteomusculares. Ante ello, emerge como pregunta norteadora: ¿Cuáles son las características del trabajo que citan los profesores como posibles factores determinan- tes para la ocurrencia de trastornos osteomusculares?

En esta perspectiva, el estudio tuvo como objetivo identificar las características de trabajo y sus implicancias en la ocurrencia de trastornos osteomusculares en profesores.

\section{MATERIAL Y MÉTODO}

Se trata de un estudio descriptivo y transversal, que abarca la temática trastorno osteomuscular acontecidos en el trabajo docente, que integra un macroproyecto de investigación intitulado "Salud, Riesgos y Enfermedades Ocupacionales - estudio integrado en diferentes ambientes de trabajo" (30). El estudio fue realizado por el Laboratorio de Estudios de Procesos Socioambientales y Producción Colectiva de Salud - LAMSA, vinculado a la Escuela de Enfermería de la Universidad Federal de Río Grande (Fundação Universidade Federal do Rio Grande) - FURG, en junio de 2012 en un pequeño municipio de la región metropolitana de Porto Alegre, Rio Grande do Sul/Brasil (39.685 habitantes). Este proyecto involucró la integración entre la Universidad Federal de Río Grande FURG y la comunidad escolar del pequeño municipio.

Todos los profesores de las escuelas de enseñanza pública infantil y fundamental fueron invitados por la Universidad y por la Secretaría Municipal de Educación a participar de un taller de integración de trabajo colectivo en salud. De esta forma se obtuvo una muestra intencional que totalizó 37 profesores. Los criterios de inclusión fueron: profesor de escuela pública; participante del taller de integración y registro de aceptación de participación en la investigación mediante firma del Término de Consentimiento Libre y Aclarado (TCLE).

Para la recolección de datos se utilizó: 1. Cuestionario autoaplicado, cerrado y de múltiple elección, que contempló variables 
relacionadas a la caracterización de los sujetos, las características ambientales de la escuela y de los trastornos osteomusculares, que fue previamente validado por el LAMSA. El cuestionario fue entregado a los participantes al inicio de las actividades del taller de integración de trabajo colectivo en salud. Todos los datos fueron recolectados por la investigadora responsable y por un grupo de académicos de Posgrado Stricto Sensu, durante el taller de trabajo en salud.

Para la organización y análisis cuantitativo de los datos se utilizó el software Statistical Package sea the Social Sciences (SPSS) versión 19.0. Este fue realizado por medio de un análisis estadístico descriptivo simple, ponderando la frecuencia absoluta y el porcentaje de los datos. Enseguida, los datos fueron agrupados en las variables correspondientes al foco del estudio -las características de trabajo y sus implicaciones para la ocurrencia de trastornos osteomuscualres.

El proyecto fue sometido al Comité de Ética en Investigación en Salud de la FURG, obteniendo la aprobación conforme el Parecer 109/2010. Los sujetos de la investigación fueron invitados a participar e informados sobre los objetivos del estudio y la garantía del anonimato, autorizando la participación por medio del Consentimiento Libre e Informado.

\section{RESULTADOS}

El análisis cuantitativo permitió la presentación de los resultados en tres categorías: caracterización de los sujetos, características organizacionales y de las condiciones de trabajo y enfermedades osteomusculares autorreferidas por los profesores.

\section{Caracterización de los participantes del estudio}

En la caracterización de los sujetos involucrados, todos eran de sexo femenino y $34(91,9 \%)$ se autoevaluaron de raza blanca. La media de edad fue de 39,6 años (DS 10,06), variando entre 19 y 68 años de edad; $27(73 \%)$ de las profesoras eran casadas o vivían en unión consensual. Con relación a la escolaridad, la mayoría 17 (45,9\%) de las profesoras posee Posgraduación completa (Tabla 1).

Tabla 1. Características demográficas de profesores actuantes en un municipio de la región metropolitana de Porto Alegre/RS, 2012.

\begin{tabular}{llcc}
\hline Variables & & $\mathbf{n}$ & \% \\
\hline Estado civil & & \multicolumn{2}{c}{18,9} \\
& Soltero & 7 & 73 \\
& Casado/unión consensual & 27 & 8,1 \\
& Separado/desquitado/divorciado & 3 & \\
Escolaridad $^{*}$ & & \multicolumn{2}{c}{10,8} \\
& Secundaria completa & 4 & 18,9 \\
& Cursos superior completo & 7 & 18,9 \\
& Posgraduación incompleto & 7 & 45,9 \\
& Posgraduación completo & 17 & \\
Cuántas escuelas/instituciones trabaja $^{*}$ & 28 & 75,7 \\
& 1 & 7 & 18,9 \\
& 2 & 2 & 5,4 \\
\hline
\end{tabular}


Continuación Tabla 1.

\begin{tabular}{crc}
\hline Tiempo de actuación en la escuela & 3 & \\
Menos de 1 año & 10 & 27 \\
De 1 a 5 años & 5 & 13,5 \\
De 5 a 10 años & 19 & 51,4 \\
Más de 10 años & & \\
Tiempo de actuación en la docencia & 1 & 2,7 \\
Menos de 1 año & 6 & 16,2 \\
De 1 a 5 años & 6 & 16,2 \\
De 5 a 10 años & 23 & 62,2 \\
Más de 10 años & 1 & 2,7 \\
Nula &
\end{tabular}

* Los números para cada elemento puede totalizar menos que $\mathrm{n}$ total debido a los valores que faltan.

\section{Características organizacionales y condiciones de trabajo}

Los sujetos citaron como factores que caracterizan el trabajo y que pueden influir en la ocurrencia de las enfermedades osteomusculares los gestos repetitivos, referidos por 11
$(29,7 \%)$ de las profesoras y el desarrollo de actividades directamente con alumnos en el período de treinta a cuarenta horas semanales, mencionado por $14(37,8 \%)$ profesoras, entre otras características presentadas en la Tabla 2.

Tabla 2. Factores organizacionales del trabajo que influencian la ocurrencia de las enfermedades osteomusculares, autorreferidas por los profesores de un municipio de la región metropolitana de Porto Alegre/RS, 2012.

\begin{tabular}{|c|c|c|}
\hline Factores organizacionales relacionados al desarrollo de LER/DORT & $\mathbf{n}$ & $\%$ \\
\hline \multicolumn{3}{|l|}{ Realización de esfuerzo físico intenso* } \\
\hline Sí & 5 & 13,5 \\
\hline No & 13 & 35,1 \\
\hline \multicolumn{3}{|l|}{ Cargar peso con frecuencia ${ }^{\star}$} \\
\hline Sí & 5 & 13,5 \\
\hline No & 16 & 43,2 \\
\hline \multicolumn{3}{|l|}{ Realización de gestos repetitivos ${ }^{*}$} \\
\hline Sí & 11 & 29,7 \\
\hline No & 16 & 43,2 \\
\hline \multicolumn{3}{|l|}{ Realización de fuerza muscular en miembros superiores ${ }^{\star}$} \\
\hline Sí & 4 & 10,8 \\
\hline No & 17 & 45,9 \\
\hline \multicolumn{3}{|l|}{ Horas semanales de trabajo directo con alumnos* } \\
\hline Menos de $10 \mathrm{~h}$ & 5 & 13,5 \\
\hline De 10 a $20 \mathrm{~h}$ & 8 & 21,6 \\
\hline De 20 a $30 \mathrm{~h}$ & 3 & 8,1 \\
\hline De 30 a $40 \mathrm{~h}$ & 14 & 37,8 \\
\hline Más de 40 h & 3 & 8,1 \\
\hline
\end{tabular}




\begin{tabular}{lrr} 
Funciones que desempeña $^{* *}$ & & \\
Enseñar & 26 & 70,3 \\
Trabajo administrativo & 6 & 16,2 \\
Coordinación de la escuela & 6 & 16,2 \\
Supervisión & 5 & 13,5 \\
Dirección & 9 & 24,3 \\
Vicedirección & 4 & 10,8 \\
Aprendiz & 1 & 2,7 \\
\hline
\end{tabular}

* Los números para cada elemento puede totalizar menos que $n$ total debido a los valores que faltan.

** Pregunta de opción múltiple.

Con relación a las condiciones de trabajo que influencian la labor docente y que pueden generar patologías osteomusculares, 22 $(59,5 \%)$ de las profesoras evalúan el espacio en sala de clases como insuficiente para su adecuado movimiento y 19 (51,4\%) de las profesoras mencionan que los muebles utilizados en el desarrollo del trabajo no son adecuados a sus estaturas. Sin embargo, 23 $(62,2 \%)$ de las participantes ponderaron que los factores del ambiente de trabajo no interfieren en su salud.

\section{Enfermedades osteomusculares - LER/ EORT referidas por los profesores}

La frecuencia de ocurrencia de los trastornos osteomusculares conforme autorreferencia de los profesores, se presenta en el Gráfico 1.

Gráfico 1. Ocurrencia de enfermedades osteomusculares conforme autorreferencia de profesores de un municipio de la región metropolitana de Porto Alegre/RS, 2012.

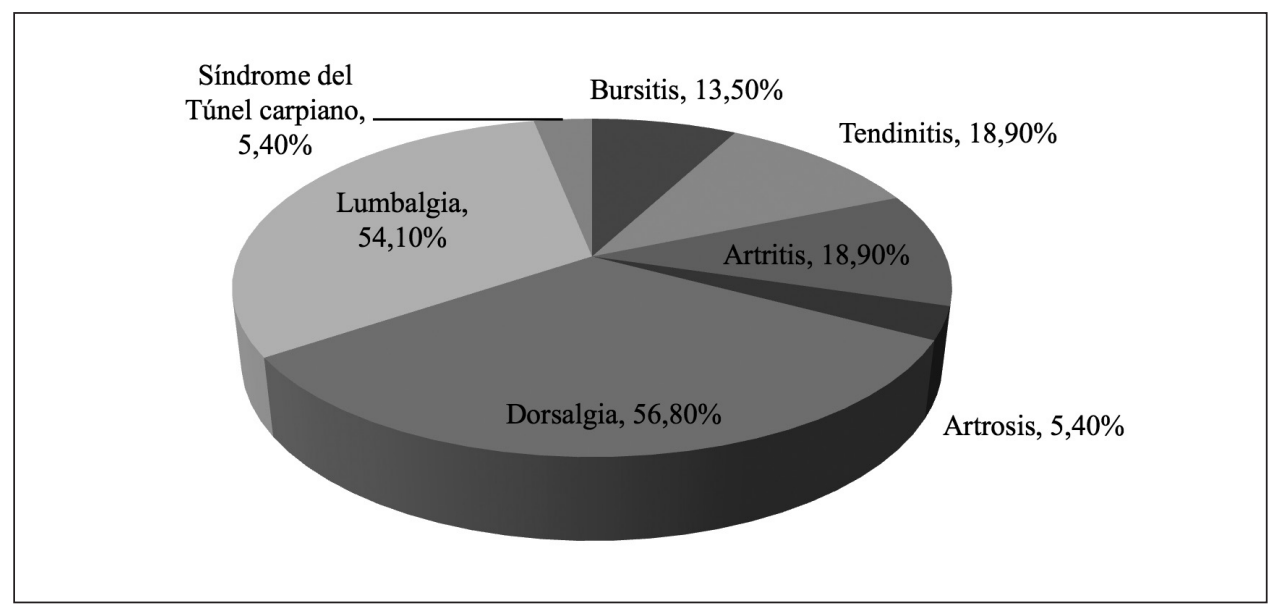

Dorsalgias y lumbalgias fueron las patologias con mayor frecuencia. Entre las profesoras que presentaron dorsalgia, $9(24,3 \%)$ re- firieron la ocurrencia en la porción cervical y $8(21,6 \%)$ en la porción torácica de la columna. De las profesoras que mencionaron 
artrosis, 1 (2,7\%) la identificó en la columna y $1(2,7 \%)$ en las rodillas. Las artritis fueron apuntadas por $7(18,9 \%)$ de las profesoras, siendo $3(8,1 \%)$ localizadas en el hombro.

En cuanto a la presentación de síntomas en el desarrollo o después del trabajo, $10(27 \%)$ de las profesoras citaron el dolor en el tronco; $26(70,3 \%)$ dolores de cabeza; $10(27 \%)$ aludieron dolor en miembros superiores y $10(27 \%)$ mencionaron dolor en miembros inferiores. Además de los datos presentados, $5(13,5 \%)$ de las profesoras refirieron calambres y adormecimiento de los miembros superiores y $10(27 \%)$ en miembros inferiores.

Además de eso, uno de los registros de las investigadoras mostró una trabajadora refiriendo presentar síndrome del túnel del carpo, expresando su dificultad con el tratamiento y la convivencia con esta patología.

\section{DISCUSIÓN Y CONCLUSIÓN}

Los resultados permiten notar la influencia, referida por las participantes del estudio -mujeres con edades entre 19 y 68 años-, de las características y condiciones del trabajo docente en la ocurrencia de trastornos osteomusculares. Cabe mencionar que la ocurrencia de síntomas y patologías estuvo presente en todas las mujeres.

La literatura presenta el desencadenamiento patológico de las LER/EORT en los diferentes grupos etarios (5), relacionándolo, en los profesores mayores, al proceso de desgaste natural del cuerpo. Entre los más jóvenes, puede estar asociado a una elevada sobrecarga de trabajo, considerando que asumen un mayor número de actividades y tareas en el inicio de la carrera, quedando, así, más expuestos a los riesgos ocupacionales del proceso de trabajo docente (31), lo que puede ocasionar a corto y largo plazo, el alejamiento del trabajo, conforme señaló un estudio que identificó a las patologías osteo- musculares como el tercer principal motivo de alejamiento ocupacional (18).

La sintomatología puede originarse de los cambios ocurridos en el sistema educacional brasileño, que acarrearon nuevas atribuciones profesionales, trayendo consigo condiciones de trabajo precarias que, consecuentemente, influenciaron la ocurrencia de los trastornos musculares esqueléticos (31).

Al relacionar la escolaridad con la ocurrencia de trastornos osteomusculares, un estudio (31) indicó que profesores con formación en nivel medio presentaban mayor sintomatología muscular esquelética en relación a aquellos que poseían enseñanza superior completa. Ello puede estar relacionado a las diferentes condiciones de trabajo en este nivel, ya que estos docentes estarían en proceso de capacitación profesional, lo que aumentaría las demandas de actividades del docente. Agregándose que, después del incremento de demanda, la sintomatología instituida puede permanecer, constituyendo así casos de LER/EORT en los profesionales que tengan formación superior y posgrado, como mostró el presente estudio.

En la caracterización del proceso de trabajo docente se considera el tiempo de trabajo en la generación de la patología, ponderando que, un período superior a diez años de actuación, contribuye a la sintomatología. El resultado fue corroborado por un estudio, que afirmó que un mayor tiempo de actividad docente está asociado al desarrollo de los trastornos en cuestión (31). Además de eso, se considera también que profesores que se desempeñan en más de una institución de enseñanza tienden a presentar trastornos osteomusculares (31); sin embargo, entre los datos se percibió la misma tendencia a la ocurrencia de síntomas y patologías entre profesores que actúan en una única institución. Ese factor se convierte en un agravante, pues destaca que la exposición en un local de trabajo es capaz de provocar el desarrollo patológico.

Con relación a las características de traba- 
jo, las profesoras señalaron la realización de esfuerzos repetitivos, que pueden estar asociados a la carga horaria de trabajo, la cual, para la mayoría de las entrevistadas, fue de treinta a cuarenta horas, representando mayor tiempo de exposición y una mayor tendencia al desarrollo patológico (31-32).

Respecto de la adecuación de los muebles en sala de clases, la mayoría de las profesoras se mostró insatisfecha, similar a los participantes de otro estudio, cuya insatisfacción con los muebles causó trastornos en la región dorsal (31). La insatisfacción también se mostró relacionada a las sillas y mesas, que impiden el adecuado posicionamiento corporal y la postura que el profesor necesita mantener para hacer uso del pizarrón (1).

Se identificó que las algias musculares surgieron tanto durante el desarrollo del trabajo y posteriormente, como consecuencia de la exposición continúa a los factores de riesgo inherentes al proceso de trabajo. En el contexto estudiado fueron señalados dolores de cabeza, en la región dorsal, en los miembros superiores e inferiores; ello fue investigado en un estudio que identificó quejas musculares en la región superior e inferior de la espalda (4) y en las regiones lumbares, cervicales y torácicas (5). Además de la citada ocurrencia, se detectó también la presencia de calambres en miembros, que puede estar relacionada a los largos períodos de pie, durante las actividades docentes.

Dichas condiciones pueden conducir al alejamiento del trabajo, pues impiden la manutención de las actividades de la vida diaria que, por consiguiente, producen otros efectos, como depresión, obesidad y cardiopatías (5).

Se identificó que la sobrecarga muscular esquelética en el trabajo de los profesores ha sido señalada en diversos estudios (1-5); sin embargo, la descripción de los tipos patológicos que afectan a estos profesionales aún es ampliamente investigado. En ese sentido, se muestra, en el presente estudio, que las lumbalgias y dorsalgias cervicales y torácicas se presentaron con alta incidencia, así como las tendinitis y artritis.

Se destaca entre las patologías la ocurrencia de la lumbalgia, la cual se visibiliza como la causa más común de limitación de la actividad en personas de diferentes rangos etarios y por ser una de las principales razones para la búsqueda de asistencia médica (33). Los factores de riesgo que más producen las lumbalgias de origen ocupacional son: la manipulación manual de materiales, el trabajo físico pesado, la flexión del tronco en movimientos de doblar y torcer, y la exposición a las vibraciones de cuerpo entero (33). En este estudio la incidencia de esta y de otras patologías osteomusculares puede indicar la necesidad de actuación sobre las condiciones de trabajo que están afectando la salud muscular de los profesores.

La frecuencia de enfermedades osteomusculares autorreferidas por las profesoras origina el planeamiento de acciones en salud para la construcción de estrategias de rehabilitación de las enfermedades osteomusculares. La enfermería puede actuar en el incentivo a la participación en grupos de la comunidad, en los que los profesores pueden problematizar la presencia patológica junto a otros individuos acometidos por la misma dolencia, considerando que, en tales ambientes, los intercambios de experiencias auxilian y estimulan al individuo a lidiar mejor con el dolor crónico. Además, tal conducta contribuye para la visualización del valor que el trabajo representa para su salud, considerando que el buen desempeño en éste debe sumarse a la salud para la producción de la satisfacción, motivación y bienestar del profesional (34).

Además, la promoción de estrategias interventoras que faciliten el desarrollo de sus actividades de rutina, la discusión de las terapéuticas más efectivas para alivio del dolor y malestar -tanto medicamentosas como no medicamentosas-; y la búsqueda de entretenimiento y actividades que den placer junto a la familia y los amigos actúan como 
incentivo a los cuidados con su salud y a los cambios que puede realizar para mejorar su bienestar en el día a día (31).

La realización del tratamiento médico convencional, únicamente, no se presenta como alternativa favorable a la solución de los cuadros de los tipos de enfermedades relacionadas al trabajo aquí discutidas. Existe la necesidad de evaluación del puesto de trabajo y, de ser necesario, la adecuación de esos ambientes, proponiendose la recuperación de los trabajadores. Además de los cambios en ese ámbito, se destaca la necesaria reducación del sujeto para el autocuidado, mediado por las pausas ocupacionales, mantención postural, alternancia entre los miembros de apoyo en el trabajo, en las posiciones de pie y sentada y la atención al mobiliario, de forma que sean adecuados a la postura correcta del trabajador.

Las profesoras fueron afectadas por distintos síntomas y patologías, surgiendo el compromiso osteomuscular a partir de su proceso de trabajo, según su autorreporte. Entre las patologías osteomusculares fueron citadas la lumbalgia, dorsalgia, artritis, artrosis, tendinitis, bursitis y el síndrome del túnel carpiano. Frente a lo expuesto, cabe resaltar que las profesoras no reconocieron la realización de esfuerzos físico y muscular intensos, pero sí la repetitividad de los movimientos y que los muebles puedan estar interfiriendo en su salud muscular, sin embargo, no consideran que los aspectos ambientales interfieran en la salud.

En virtud de ello, se constata el conocimiento proporcionado por la investigación como indicativo de que la enfermería puede actuar frente a los riesgos y agravios ambientales que afectan a grupos de población específicos, como es el caso de la salud del profesor. Así, sus acciones en salud tendrán mayor eficacia al planear estrategias de prevención e intervención, con vistas a la minimización de los daños causados por LER/EORT. De ese modo, el enfermero actúa en la investigación, promoción de la salud y prevención de los riesgos y agravios que afectan a los profesores, debido a su proceso y ambiente de trabajo.

Se presenta como limitación de este estudio el número reducido de sujetos, lo que restringe la posibilidad de generalización de los resultados obtenidos. Sin embargo, se presenta una realidad de trabajo amplia para actuación de el/la enfermero/a, lo que remite a la sugerencia de que estudios con este abordaje sean replicados con otros profesores, a fin de visibilizar la ocurrencia sintomatológica y patológica en diferentes procesos de trabajo en la enseñanza.

\section{REFERENCIAS}

1. Lima MFEM, Lima-filho DO. Condições de trabalho e saúde do/a professor/a universitário/a. Ciênc cogn. 2009; 14(3): 62-82.

2. Chiu TTW, Lam PKW. The prevalence of and risk factors for neck pain and upper limb pain among secondary school teachers in Hong Kong. J Occup Rehabil. 2007; 17(1): 19-32.

3. Aguayo AT, Klijn TP. Morbilidad de la mujer trabajadora, servicio de salud Concepción, Chile. Cienc enferm. 2005; $\mathrm{XI}(1)$ : 73-84.

4. Fernandes MH, Rocha VM, Costa-Oliveira AGR. Fatores associados à prevalência de sintomas osteomusculares em professores. Rev. salud pública. 2009; 11(2): 256-67.

5. Carvalho AJFP, Alexandre NMC. Sintomas osteomusculares em professores do ensino fundamental. Rev bras fisioter. 2006; 10(1): 35-41.

6. Erick PN, Smith DR. A systematic review of musculoskeletal disorders among school teachers. BMC Musculoskelet Disord. 2011; 12: 260.

7. Yue P, Liu F, Li L. Neck/shoulder pain and low back pain among school teachers in 
China: prevalence and risk factors. BMC Public Health. 2012; 12: 789.

8. Santos GLV, Silva IL, Cardoso F, Beresford H. Ocorrência de distúrbios osteomusculares relacionados ao trabalho de professores de uma instituição de ensino superior de Belém/PA. Fisioter Bras. 2009; 10(4): 263-9.

9. Coelho CT, Dias D, Neto M, Matos MA. Prevalência da síndrome do ombro doloroso (SOD) e sua influência na qualidade de vida em professores de uma instituição privada de nível superior na cidade de Lauro de Freitas, Bahia. Rev baiana saúde pública. 2010; 34(Suppl 1): 19-29.

10. Antonelli BA, Xavier AAP, Oenning P, Baumer MH, Silva TFA, Pilatti LA. Prevalence of cervicobrachial discomforts in elementary school teachers. Work. 2012; 41(1): 5709-14.

11. Araújo TM, Carvalho FM. Condições de trabalho doecente e saúde na Bahia: estudos epidemiológicos. Educ Soc. 2009; 30(107): 427-49.

12. Porto LA, Reis IC, Andrade JM, Nascimento CR, Carvalho FM. Doenças ocupacionais em professores atendidos pelo Centro de estudos da Saúde do Trabalhador (CESAT). Rev baiana saúde pública. 2004; 28(1): 33-49.

13. Ribeiro IQB, Araújo TM, Carvalho FM, Porto LA, Reis EJFB. Fatores ocupacionais associados á dor musculoesquelética em professores. Rev baiana saúde pública. 2011; 35(1): 42-64.

14. Ministério da Saúde (Brasil). Organização Pan-Americana da Saúde no Brasil. Doenças relacionadas ao trabalho: manual de procedimentos para os serviços de saúde. Brasília: Ministério da Saúde; 2001.

15. Díaz MCM, Marziale MHP, Robazzi MLCC, Freitas FCT. Lesiones osteomusculares en trabajadores de un hospital mexicano y la ocurrencia del ausentismo. Cienc enferm. 2010; XVI(2): 35-46.

16. Araújo TM, Sena IP, Viana MA, Araújo
EM. Mal-estar docente: avaliação de condições de trabalho e saúde em uma instituição de ensino superior. Rev baiana saúde pública. 2005; 29(1): 6-21.

17. Assunção AA, Oliveira DA. Intensificação do trabalho e saúde dos profesores. Educ Soc. 2009; 30(107): 349-72.

18. Gasparini SM, Sandhi MB, Assunção AA. O professor, as condições de trabalho e os efeitos sobre sua saúde. Educação e Pesquisa. 2005; 31(2): 189-99.

19. Godoy SCB, Alves M, Rocha ADEM, Santana D. Work absence by disease in a public hospital net. A descriptive study. Online Braz J Nurs [Internet]. 2006 [citado 06 dic 2012]; 5(3). Disponible en: http:// www.objnursing.uff.br/index.php/nursing/article/view/544/123

20. Almeida MCV, Cezar-Vaz MR, Soares JFS, Silva MRS. The prevalence of musculoskeletal diseases among casual dock workers. Rev Lat Am Enfermagem. 2012; 20(2): 243-50.

21. Murofuse NT, Marziale MHP. Mudanças no trabalho e na vida de bancários portadores de lesões por esforços repetitivos: LER. Rev Lat Am Enfermagem. 2001; 9(4): 19-25.

22. Santos Júnior AV, Mendes AM, Araújo LKR. Experiência em clínica do trabalho com bancários adoecidos por Ler/Dort. Psicol ciênc prof. 2009; 29(3): 614-25.

23. Chowanadisai S, Kukiattrakoon B, Yapong B. Occupational health problems of dentists in southern Thailand. Int Dent J. 2000; 50(1): 36-40.

24. Innes E, Walsh C. Musculoskeletal disorders in Australian dairy farming. Work. 2010: 141-55.

25. Osborne A, Blake C, McNamara J, Meredith D, Phelan J, Cunningham C. Musculoskeletal disorders among Irish farmers. Occup Med. 2010; 60: 598-603.

26. Jakob M, Liebers F, Behrendt. The effects of working height and manipulated weights on subjective strain, body posture and muscular activity of milking par- 
lor operatives - Laboratory study. Appl Ergon. 2012; 43: 753-61.

27. Douphrate DI, Fethke NB, Nonnenmann MW, Rosecrance JC, Reynolds SJ. Full shift arm inclinometry among dairy parlor workers: a feasibility study in a challenging work environment. Appl Ergon. 2012; 43: 604-13.

28. Patil A, Rosecrance J, Douphrate D, Gilkey D. Prevalence of carpal tunnel syndrome among dairy workers. Am J Ind Med. 2012; 55: 127-35.

29. Reis EJFB, Carvalho FM, Araújo TM, Porto LA, Silvany Neto AM. Trabalho e distúrbios psíquicos em professores da rede municipal de Vitória da Conquista, Bahia, Brasil. Cad Saude Publica. 2005; 21(5): 1480-90.

30. Cezar-Vaz MR. Saúde, Riscos e Doenças ocupacionais: estudo integrado em diferentes ambientes de trabalho. Universidade Federal do Rio Grande: Rio Grande (RS), Brasil, 2010.
31. Cardoso JP, Ribeiro IQB, Araújo TM, Carvalho FM, Reis EJFB. Prevalência de dor músculo-esquelética em professores. Rev bras epidemiol. 2009; 12(4): 604-14.

32. Cardoso JP; Araújo TM; Carvalho, FM Oliveira NF; Reis, EJF. Aspectos psicossociais do trabalho e dor músculo-esquelética em professores. Cad Saude Publica. 2011; 27(8): 1498-1506.

33. Pittsburgh, PA: U.S. Reducing Low Back Pain and Disability in Mining. Department of Health and Human Services, Public Health Service, Centers for Disease Control and Prevention, National Institute for Occupational Safety and Health, DHHS (NIOSH), Information Circular 9507. 2008 Sep; 77 p.

34. Calvo-Salguero A, Aguilar Luzon MC, Salinas JMML. Valores laborales individualistas y colectivistas de enfermeras y enfermeros en un entorno multicultural. Index enferm. 2008; 17(4): 246-50. 